P4133

\title{
Atrial fibrillation in elderly patients with syncope and dementia: clinical insights from a large multicenter Italian registry
}

\author{
A. Ceccofiglio ${ }^{1}$, S. Fumagalli ${ }^{1}$, E. Mossello ${ }^{1}$, C. Mussi ${ }^{2}$, M. Bo $^{3}$, A.M. Martone ${ }^{4}$, G. Bellelli ${ }^{5}$, F. Nicosia ${ }^{6}$, D. Riccio ${ }^{7}$, A. Langellotto ${ }^{8}$, G. Tava $^{9}$, \\ V. Boccardi ${ }^{10}$, E. Tonon ${ }^{1}$, P. Abete ${ }^{11}$, A. Ungar ${ }^{1}$ \\ ${ }^{1}$ University of Florence, Florence, Italy; ${ }^{2}$ University of Modena \& Reggio Emilia, Modena, Italy; ${ }^{3}$ Hospital Citta Della Salute e della Scienza di \\ Torino, Turin, Italy; ${ }^{4}$ Catholic University of the Sacred Heart, Rome, Italy; ${ }^{5}$ University of Milan-Bicocca, Monza, Italy; ${ }^{6}$ Civil Hospital of Brescia, \\ Brescia, Italy; ${ }^{7}$ University of Cagliari, Cagliari, Italy; ${ }^{8}$ Hospital Santa Maria di Ca Foncello, Treviso, Italy; ${ }^{9}$ Santa Chiara Hospital in Trento, Trento, \\ Italy; ${ }^{10}$ Hospital Santa Maria Della Misericordia, Perugia, Italy; ${ }^{11}$ Federico II University Hospital, Naples, Italy
}

Funding Acknowledgement: None

Background: Syncope and dementia have a high prevalence in elderly individuals. Atrial fibrillation (AF) frequently occurs at advanced age. The coexistence of these conditions can be indicative of a clinically relevant frail status.

Purpose: The aim of this study was to evaluate the characteristics and the long-term outcome of AF patients with dementia and a history of syncope. Methods: We evaluated the Syncope and Dementia (SYD) Registry. Data were collected by 11 Geriatric Departments between 2012 and 2016 . Follow-up was closed at the 12-month evaluation.

Results: During the study period, 522 patients (women - 324, 62.1\%; MMSE: $17 \pm 6$ ) were enrolled. Of these $138(26.4 \%)$ have or presented a history of AF. Patients with AF were older ( $85 \pm 6$ vs. $83 \pm 6$ years, $p=0.012$ ), with a higher heart rate $(78 \pm 17$ vs. $73 \pm 14 \mathrm{bpm}, \mathrm{p}<0.001)$, had a more complex clinical picture with an increased number ( $3.9 \pm 2.0$ vs. $3.0 \pm 1.8$, $p<0.001)$ and severity $(1.8 \pm 0.3$ vs. $1.6 \pm 0.4, p<0.001)$ of comorbidities, as assessed with the Cumulative Illness Rating Scale. In particular, the prevalence of diabetes ( 28.3 vs. $20.1 \%, p=0.047$ ), heart failure (13.8 vs. $7.3 \%, \mathrm{p}=0.023)$ and stroke/TIA ( 26.1 vs. $17.7 \%, \mathrm{p}=0.035)$ was higher in patients with the arrhythmia. Cardiac syncope was more frequently diag- nosed at the final evaluation (18.8 vs. $4.9 \%, p<0.001)$. Even if the use of antipsychotics ( 13.0 vs. $27.6 \%, p=0.001$ ) and cholinesterase inhibitors (6.5 vs. $16.4 \%, p=0.004$ ) were less used in $A F$ subjects, the total number of prescribed drugs was higher $(6.9 \pm 2.9$ vs. $5.9 \pm 2.7, p<0.001)$. At multivariate analysis (overall predictivity: $75 \%$ ), AF patients were characterized by advanced age $(p=0.041)$, a higher severity of comorbidities $(p<0.001)$, a greater number of drugs $(p=0.020)$, an increased heart rate $(p=0.004)$ and a more frequent presence of cardiac symptoms $(p=0.049)$.

At one-year follow-up (8 patients lost), the mortality rate in AF patients was $27.7 \%(\mathrm{~N}=36 / 130)$. Deceased patients presented a greater degree of disability (number of lost activities of daily living: $3.7 \pm 2.3$ vs. $2.8 \pm 1.9, p=0.020$ ) and a higher heart rate at baseline ( $85 \pm 17$ vs. $76 \pm 15 \mathrm{bpm}, \mathrm{p}=0.006)$. Multivariate analysis (overall predictivity: $74 \%$ ) confirmed the association of disability $(\mathrm{p}=0.039)$ and heart rate $(\mathrm{p}=0.045)$ with prognosis.

Conclusions: $\mathrm{AF}$ is frequently present in patients with dementia and a history of syncope. It is usually associated with a more complex clinical picture and high long-term mortality. Heart rate and a higher degree disability seem to be related to prognosis. 